\title{
eDrive-ready telemetry systems for rotating applications
}

\author{
Gerhard Spitz, Technical Director \\ AXON Systems GmbH \\ Wildmoos 5, 82266 Inning am Ammersee, Germany \\ g.spitz@axon-systems.com
}

\section{Abstract:}

Telemetry systems for rotating installations providing contactless data transmission is state of the art in many branches of mechanical industry. The measurement of physical quantities on rotating vehicle and machine components is an important component in the development of modern drives.

Inductive power supply in combination with RF data transmission ensures contact-free and therefore wear-free operation. In addition to the anyway challenging environment, modern drive concepts requiring latest telemetry systems to be adapted to some new challenges, especially strong EMC impacts in the surrounding of the electric engine.

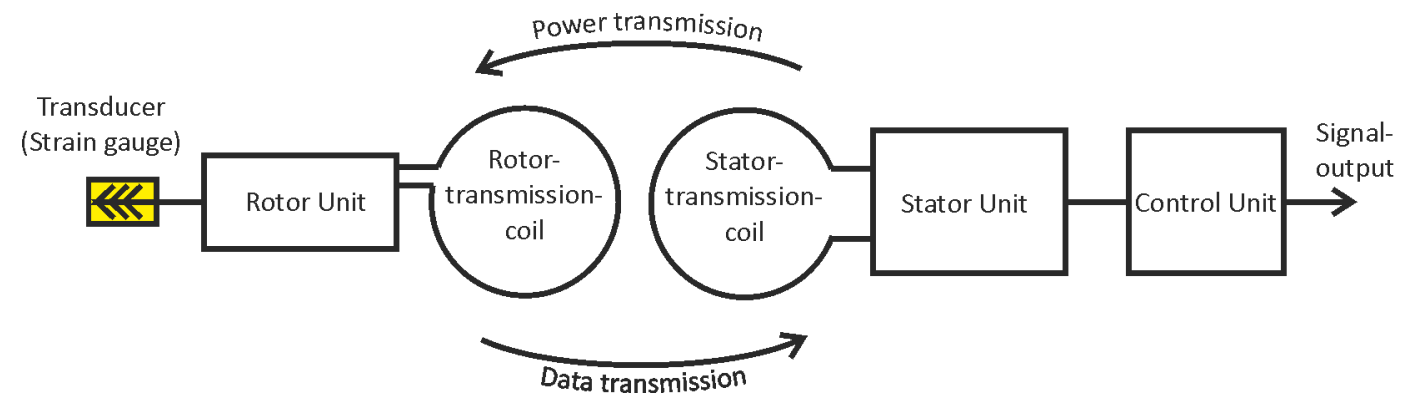

Telemetry systems for rotating installations consisting of:

Rotor Unit

Sensor supply

Signal acquisition and processing

RF Data transmission

Stator Unit

Inductive power supply

RF Data reception 


\section{Control Unit}

Central processing unit

RF reception, decoding

Signal playback

Inductive power control
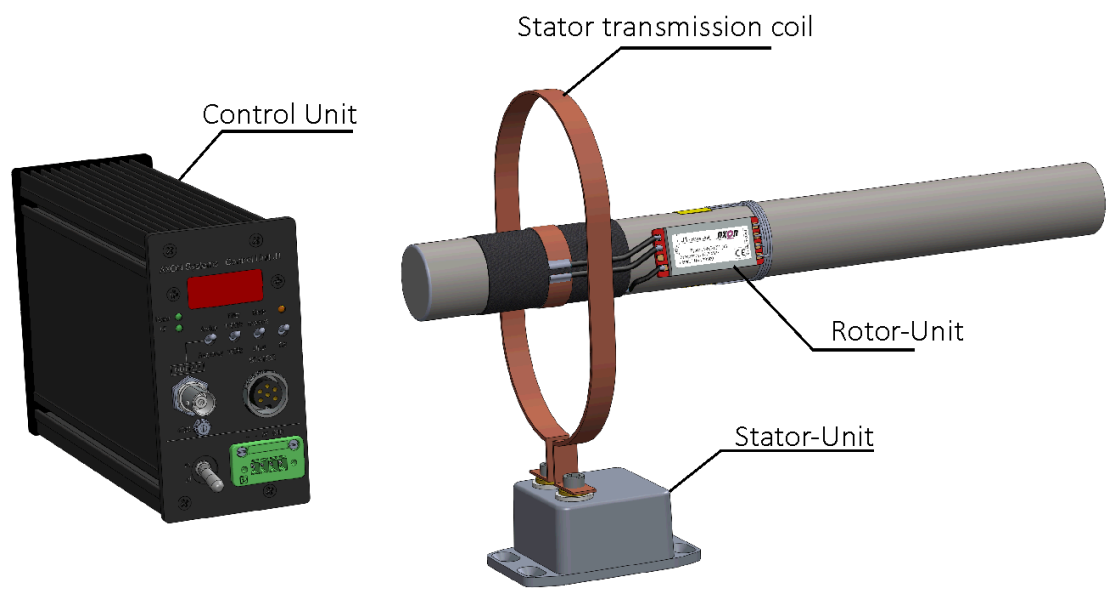

A classic powertrain, containing a motor, a gearbox, propellershaft and 2 or even 4 half shafts can require many different torque-, force- and temperature-measurements on its rotating parts. Certainly, the most common telemetry measurement for automotive applications is measuring torque on drive shafts during test drives and long-term testing. But also, the gearbox input, crankshaft or cardan shafts necessitating torque measurements. Temperature measurements for example can be necessary on brake discs, clutch- and differential plates or even single gearings.

Highest possible efficient drivetrains are going to be increasingly important, what also makes the constructions complexity enormous increasing.

\section{Particularities on eDrive applications:}

High packaging density

High operating temperature

High RPM

High EMC load

Electric engines in Automotive applications coming with extremely high power, controlled by steep edges which leads to immense EMC load on the drivetrain. Shafts might even work as "transmitting antennas" for the disturbances. As for example torque or temperature measurements are based on transducer signals in the range of $\mu \mathrm{V}$, it is obvious that these environmental conditions can lead to signal spikes or even total data loss. 
As packing density in mechanical technology is increasing, installation space for additional measuring technology or telemetry systems is continually shrinking. Telemetry needs to be both, small and flexible in its installation to use remaining installation space in an optimal way.

\section{Meet the requirements of modern eDrive applications}

Rotor Units of latest telemetry systems needs to be equipped with an extreme powerful RF transmitter by meeting the RTT\&T-standards coming with the CE-certification at the same time. Also, the Control Unit is coming with a new, extreme sharp filter for best possible data reception.

Stator-Units with option "E" additionally are equipped with an EMC suppression right on the receiving antenna which allows an early as possible filtering of any disturbing fields. The inductive power transmission of the Stator Unit is not affected and remains extremely powerful. Besides EMCinterferences also the size of the telemetry components needs to be considered. The arrangement of Rotor Unit, Stator Unit, antenna and transducer needs to be possible in different ways to use the space in an optimal way. Rotor Units are available in different designs in order to meet the requirements of any individual application. Electronics are available in rectangular shape with aluminium housing, round in carbon housing or flexible without housing to be "wrapped" around the shaft.

The Control Unit works as the central processing unit, controlling the inductive power supply and the data reception. It allows to be different Stator Units connected as well as shaft diameters between $5 \mathrm{~cm}$ to 2 meters.

As modern drivetrains can develop a lot of torque, particularly while misuse-testing half shafts can break. To avoid losing a telemetry installation if a shaft breaks, the torque measurement can be carried out on gearbox output flanges as these components are even more robust against torque peaks. A broken half shaft can be easily replaced, and the torque measuring device remains undamaged.

Gearbox output flanges as well as shaft installations with a measuring range of just a few $\mathrm{Nm}$ as well as even $\mathrm{MNm}$ - all can be operated with the same system without the need to change any adjustments.

To arrange a torque measuring installation, no mechanical manipulation on the test object is needed. Strain gauges, telemetry, antenna and wiring are bonded to the shaft by using advanced adhesive technologies.

The same applies for temperature measurements. Full flexibility allows measurements in electric engines, clutch plates, joints, gearings and many more. Latest systems can measure up to $1.250^{\circ} \mathrm{C}$. Those systems are especially designed for measuring temperatures on brake discs, such as necessary on dynamometer test benches. When used in big test benches, EMC will be an issue as the test stands are usually equipped with large frequency converters which can affect electrical installations due to EMC impacts. Temperature telemetries with "option E" allow safe and reliable measurements in this difficult environment. 